\title{
Vibrational analysis of peptides, polypeptides and proteins
}

XXVII. Structure of gramicidin S from normal mode analyses of low-energy conformations.

\author{
V.M. NAIK' ' S. KRIMM' ' J.B. DENTON ${ }^{2}$, G. NÉMETHY ${ }^{2}$ and H.A. SCHERAGA ${ }^{2}$ \\ ${ }^{1}$ Biophysics Research Division, University of Michigan, Ann Arbor, MI, and \\ ${ }^{2}$ Baker Laboratory of Chemistry, Cornell University, Ithaca, NY, USA
}

Received 3 May, accepted for publication 17 May 1984

\begin{abstract}
Normal mode calculations have been carried out on three low-energy structures of gramicidin S obtained from conformational energy calculations. When the results on the amide modes are compared with observed bands in the infrared and Raman spectra of crystalline gramicidin $S$ and its $N$-deuterated derivative, one of the structures is clearly disfavored. Of the other two, one is slightly favored, and it corresponds to the lowest-energy structure obtained from the energy calculations. Spectra from solutions in DMSO and $\mathrm{CH}_{3} \mathrm{OH}$ suggest that the molecular conformation is essentially retained in these solvents.
\end{abstract}

Key words: gramicidin S; infrared spectra; normal mode calculations; Raman spectra; $\beta$ turns

Normal mode calculations provide a powerful method for interpreting the infrared (i.r.) and Raman spectra of peptide molecules in terms of their conformations (1). This is especially true if the number of likely structural possibilities can be limited by conformational energy calculations $(2,3)$. In such a case, normal mode calculations for each of the structures can be compared with the observed vibrational spectra, thereby making possible a more definitive structural assignment. In this paper we report such a study for gramicidin $\mathbf{S}$ (GrS).

The GrS molecule is a cyclosymmetric decapeptide antibiotic whose chemical structure is (L-Val-L-Orn-L-Leu-D-Phe-L-Pro) ${ }_{2}$. Early $X$-ray crystallographic studies (4) indicated the presence of a two-fold axis of symmetry, and led to the proposal of a $\beta$-sheet model as one of the most probable structures (5). A similar structure was proposed on the basis of n.m.r. studies (6). It consists of two antiparallel Val-Orn-Leu strands connected by type II $^{\prime}$ $\beta$-turns (7) formed by the D-Phe-Pro residues, the structure being stabilized by four intramolecular hydrogen bonds between $\mathrm{NH}$ and $\mathrm{CO}$ groups of Val and Leu. A subsequent n.m.r. study aimed at deriving the dihedral angles (8) led to the suggestion of a type III $\beta$-turn for the connecting residues, and such a structure was also obtained from early empirical conformational energy calculations (9). However, another analysis of the n.m.r. data in conjunction with i.r. studies of the NH stretch modes favored the type $\mathrm{II}^{\prime} \beta$-turn (10), and this structure was further supported by a study involving empirical energy calculations combined with an analysis of experimental data (11).

In a systematic and extensive conformational energy study of symmetric GrS molecules with exact ring closure (12), the type II' $\beta$-turn structure was shown to be the most stable. This 
work predicted three such similar structures, M1, M2, and M3, with M1 (of lowest energy, $-14.4 \mathrm{kcal} / \mathrm{mol}$ ) being about $2 \mathrm{kcal} / \mathrm{mol}$ more stable than the other two (of energy - 12.3 and $-12.2 \mathrm{kcal} / \mathrm{mol}$, respectively). The $\mathrm{M} 1$ structure was in agreement with available experimental data, and more recently has been shown $(13,14)$ to be very similar to the structure of a hydrated GrS-urea complex derived from a single crystal $X$-ray analysis (15). Small differences in conformation at the $\mathrm{Val}$ and Orn residues (with the retention of an approximate two-fold axis of symmetry in the complex) were attributed to intermolecular interactions in the crystal (13).

Previous i.r. studies have concentrated on deriving structural information from isolated features of the spectra. Thus, an early study of the dichroism of the amide I band of single crystals of a GrS derivative (16) attempted to define the chain conformation, but arrived at the conclusion that the structure is based on a folded chain not involving a $\beta$-sheet structure. From the relative intensities of the amide I and amide II modes it was stated that the peptide groups are trans (17). Integrated intensities of $\mathrm{NH}$ stretch modes were used to determine the number of hydrogen bonded $\mathrm{NH}$ groups (10). Studies of GrS oriented in a stretched polyoxyethylene film (18) led to the conclusion that molecules associate with each other to form a "cross $\beta$ " structure, a feature noticed (13) in the X-ray structure (15). A recent careful study of the $\mathrm{NH}$ stretch modes of variably deuterated GrS molecules (19) was used to identify the bands associated with particular NH groups and, from their frequencies, to determine their hydrogen bond lengths. Raman studies have been of a similar selective nature. For example, a study of solid GrS attempted to identify $\beta$-turns from the frequencies of several amide modes (20).

In the present work, we have aimed at a more complete analysis of the vibrational spectrum of GrS. Since its structure can be thought of as a mixture of $\beta$-sheet and $\beta$-turn conformations, such a study could proceed from previous normal mode calculations of standard $\beta$-sheet (21) and $\beta$-turn (22-24) structures. However, interactions between such joined structures may not be negligible (23), and we have therefore chosen to calculate the normal modes of the entire molecule. This also makes it possible, as indicated above, to test whether the spectra can distinguish between similar structures of comparable energies (12), viz., between $M 1, M 2$, and $M 3$. As will be seen, this is indeed possible, once again demonstarting the power of normal mode calculations in providing definitive information on peptide conformation.

\section{EXPERIMENTAL PROCEDURES}

Gramicidin S was obtained from Sigma and recrystallized twice from $50 \%$ acetone. Deuterated GrS (GrS-ND) was prepared by dissolving the peptide in an excess of $\mathrm{D}_{2} \mathrm{O}$, allowing exchange for $\sim 48 \mathrm{~h}$, and lyophilizing.

Raman spectra of the crystals were recorded using a Spex 1403 double monochromator and the $514.5 \mathrm{~nm}$ excitation line of an $\mathrm{Ar}^{+}$laser. The incident power was $\sim 140 \mathrm{~mW}$ and the spectral band pass was $2 \mathrm{~cm}^{-1}$. Infrared spectra were recorded in $\mathrm{KBr}$ disks, using a Digilab FTS20C FTIR spectrometer with a resolution of $2 \mathrm{~cm}^{-1}$. Solution Raman spectra were recorded with an instrument described previously $(25,26)$, using the $488.0 \mathrm{~nm}$ line of an $\mathrm{Ar}^{+}$laser at an incident power of $150-200 \mathrm{~mW}$ and an instrumental resolution of $4 \mathrm{~cm}^{-1}$.

\section{NORMAL MODE CALCULATIONS}

The GrS structures used in the normal mode calculations had the backbone dihedral angles of the M1, M2, and M3 conformations (12), but the side chains (including the Pro ring) were approximated by point masses equivalent to $\mathrm{CH}_{3}$. This was necessary in order to make the calculation manageable, but we have already shown that such an approximation reproduces very well the non-side-chain frequencies and normal modes of a full calculation for $\alpha$ - and $\beta$-poly(L-alanine) (27). It should, therefore, provide a satisfactory basis for analyzing the backbone conformationdependent amide modes of GrS. Various parameters of these structures are given in Table 1, and a projection of $M 1$ along the two-fold symmetry axis is shown in Fig. 1. It might be 
Vibrational analysis of peptides, polypeptides and proteins

TABLE 1

Structural parameters of conformations of gramicidin $S^{\mathbf{a}}$

\begin{tabular}{|c|c|c|c|c|c|c|c|}
\hline & & $\phi$ & $\psi$ & $\mathrm{r}(\mathrm{H} \ldots \mathrm{O})$ & $\mathrm{r}(\mathrm{N} \ldots \mathrm{O})$ & $\Varangle \mathrm{NHO}$ & $\varangle \mathrm{HNO}$ \\
\hline \multirow[t]{3}{*}{ Val: } & $M 1^{b}$ & -90 & 100 & $2.32^{\mathrm{c}}$ & $3.04^{\mathrm{c}}$ & $128^{c}$ & $37^{c}$ \\
\hline & M2 & -91 & 100 & 2.23 & 2.99 & 132 & 34 \\
\hline & M3 & -97 & 127 & 3.75 & 4.33 & 120 & 49 \\
\hline \multirow[t]{3}{*}{ Orn ${ }^{d}:$} & M1 & -127 & 125 & & & & \\
\hline & M2 & -123 & 124 & & & & \\
\hline & M3 & -80 & 113 & & & & \\
\hline \multirow[t]{3}{*}{ Leu: } & M1 & -156 & 117 & $1.90^{\mathrm{e}}$ & $2.89^{\mathrm{e}}$ & $164^{e}$ & $11^{\mathbf{e}}$ \\
\hline & M2 & -152 & 114 & 1.92 & 2.89 & 165 & 10 \\
\hline & M3 & -106 & 95 & 3.13 & 3.77 & 123 & 44 \\
\hline \multirow[t]{3}{*}{ D-Phe ${ }^{d}$ : } & M1 & 60 & -137 & & & & \\
\hline & M2 & 59 & -137 & & & & \\
\hline & M3 & 92 & -148 & & & & \\
\hline \multirow[t]{3}{*}{ Prod: } & M1 & -75 & -18 & & & & \\
\hline & M2 & -75 & -13 & & & & \\
\hline & M3 & -75 & -36 & & & & \\
\hline
\end{tabular}

$\mathrm{r}$ in $\mathrm{A}$, angles in degrees.

bM1, M2, and M3 from ref. 12.

c NH(Val) ... OC(Leu) hydrogen bond.

${ }^{d}$ All external hydrogen bonds have $\mathrm{r}(\mathrm{H} \ldots \mathrm{O})=1.90 \AA, \mathrm{r}(\mathrm{N} \ldots \mathrm{O})=2.86 \AA, \varangle \mathrm{NHO}=\varangle \mathrm{COH}=160^{\circ}(\mathrm{see}$ ref. 22).

e NH(Leu) . . OC(Val) hydrogen bond.

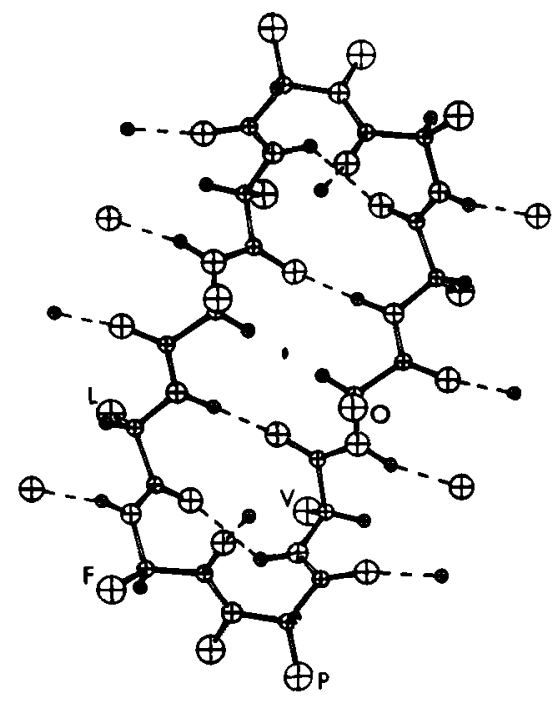

FIGURE 1

Projection of Ml structure of gramicidin S along twofold axis, showing external hydrogen bonds and point mass representation of side chains. Letters represent amino acid residue. Prolyl ring represented by $C^{\beta}$ and $C^{\delta}$ atoms (see text). noted that the backbone dihedral angles of $\mathrm{M} 1$ and $M 2$ are very similar to each other whereas they differ significantly from those of M3.

Two of the structures, $M 1$ and $M 2$, have intramolecular $\mathrm{NH}(\mathrm{Val}) . . \mathrm{OC}(\mathrm{Leu})$ and $\mathrm{NH}$ (Leu) . . . OC(Val) hydrogen bonds, whose properties are given in Table 1; both of the $r(N \ldots .0)$ for $M 3$ are so long that these hardly qualify as hydrogen bonds. The other $\mathrm{NH}$ and $\mathrm{CO}$ groups form external hydrogen bonds, and we have accounted for their influence, as before (22), by bonding an $\mathrm{O}$ atom to an $\mathrm{NH}$ group and an $\mathrm{H}$ atom to a $\mathrm{CO}$ group (the parameters are given in Table 1). This should provide a reasonable basis for determining the intramolecular frequencies, which should be influenced most by the covalent ring and intramolecular hydrogen-bonding interactions. Because the crystal structure of the GrS-urea complex (15) shows that two adjacent molecules are hydrogen bonded through the Orn NH and $\mathrm{CO}$ groups, we have assumed this to be the case for crystalline GrS and have allowed for 
this perturbation to the Orn amide $I$ and amide II modes (see below), but not in the calculation of the frequencies of a single GrS molecule.

The internal and local symmetry coordinates were defined as in earlier work (28). As a result of the two-fold symmetry axis in the molecule, there are $118 \mathrm{~A}$ species modes (symmetric with respect to this axis) and $116 \mathrm{~B}$ species modes (antisymmetric with respect to this axis), all of which can be i.r. and Raman active. However, because of the rough planarity of the molecule, we would expect strong Raman bands to be of $A$ species and strong i.r. bands to be of B species.

The force field was one recently developed for $\beta$-poly (L-alanine) with the side chain taken as a point mass (27). The intramolecular $\mathrm{H}$... O force constants for $\mathrm{M} 1$ and $\mathrm{M} 2$ were obtained by interpolation from the values for polyglycine I (29) and $\beta$-poly(L-alanine) (21), using the actual $\mathrm{r}(\mathrm{N}$. . . O) distances; for M3 these force constants were set equal to zero.

Transition dipole coupling $(30,31)$ (TDC) was incorporated, using transition moments of $\Delta \mu_{\text {eff }}=0.37 \mathrm{D}$ for amide I and $\Delta \mu_{\text {eff }}=0.269 \mathrm{D}$ for amide II. This was done in two stages. First, interactions within a single molecule were considered, taking appropriate account of the eigenvector components from the normal mode analysis. Second, since the Orn modes were essentially isolated, the interaction across these intermolecular hydrogen bonds was calculated. This TDC interaction was computed using the GrS-urea complex structure (15), the same intermolecular relationship being used for all three conformations.

\section{RESULTS AND DISCUSSION}

The Raman spectra of normal and $N$-deuterated crystalline GrS are given in Fig. 2 and the i.r. spectra of these molecules are given in Fig. 3. Raman spectra in DMSO-d $\mathrm{d}_{6}$ and in $\mathrm{CH}_{3} \mathrm{OH}$ are shown in Fig. 4. No dependence on concentration (from $2 \%$ to $9 \%, \mathrm{w} / \mathrm{v}$ ) was observed in these spectra. Since we are concerned primarily with the conformation of this molecule, and the normal mode calculations have been carried out in the side-chain point-mass approximation, we shall not attempt a complete assignment of the vibrational spectrum. Rather, we will analyze only the conformationally sensitive amide I, II, III, and V bands, the observed and calculated frequencies and potential energy distributions (PED) of which are given in Tables $2,3,4$, and 5 , respectively. Such assignment assumes that bands due to side chains are independently identifiable. This has been achieved by comparison with the detailed calculation and analysis of the $\beta$-turn structure of Pro-LeuGly- $\mathrm{NH}_{2}$ (32) for the Pro and Leu (as well as $\mathrm{Val} \mathrm{CH}_{3}$ ) modes, and with the Raman bands of Phe (33) and the Raman and i.r. spectra of polystyrene (34) for the Phe modes. Even though the structures are not identical, the identification of the $\mathrm{NH}_{2}$ modes of Orn is assisted by the Pro-Leu-Gly- $\mathrm{NH}_{2}$ analysis (32).

\section{Crystalline gramicidin $S$}

Amide I region. The amide I (mainly $\mathrm{CO}$ stretch) modes in the Raman spectra of GrS are observed at 1670VS, 1658sh, and 1646sh $\mathrm{cm}^{-1}$ (the weak shoulder near $1700 \mathrm{~cm}^{-1}$ may also be assignable to amide I), In GrS-ND the $1670 \mathrm{~cm}^{-1}$ band has shifted to $1662 \mathrm{~cm}^{-1}$ and broadened significantly (possibly showing peaks at 1662 and $1658 \mathrm{~cm}^{-1}$ ), and a shoulder is still present at $1644 \mathrm{~cm}^{-1}$. In the i.r. spectrum there is a very strong band at $1643 \mathrm{~cm}^{-1}$ which hardly shifts on $N$-deuteration, and a shoulder at $1655 \mathrm{~cm}^{-1}$ which seems to weaken in GrS-ND. [As indicated by the intensity of the amide II mode at $1534 \mathrm{~cm}^{-1}$ (see Fig. 3b), the GrS-ND is only partially deuterated. However, the evidence from the amide III region (see below) indicates that there is no differential exchange of the different $\mathrm{NH}$ groups, as occurs at lower $\mathrm{pH}$ (19).] There is probably also a shoulder near $1700 \mathrm{~cm}^{-1}$. These bands are listed in Table 2, with suggested assignments that are discussed below.

The calculated frequencies shown in Table 2 were arrived at, as indicated above, by 1) computing the normal modes of the molecule, 2) adding the intramolecular TDC contribution, and 3) then adding the intermolecular TDC contribution resulting from the hydrogen bonding of two molecules through Orn residues. The $A$ and $B$ species modes of the molecule before applying any TDC interactions were found from the calculation to be at essentially the same frequency. For example, the Orn 
Vibrational analysis of peptides, polypeptides and proteins

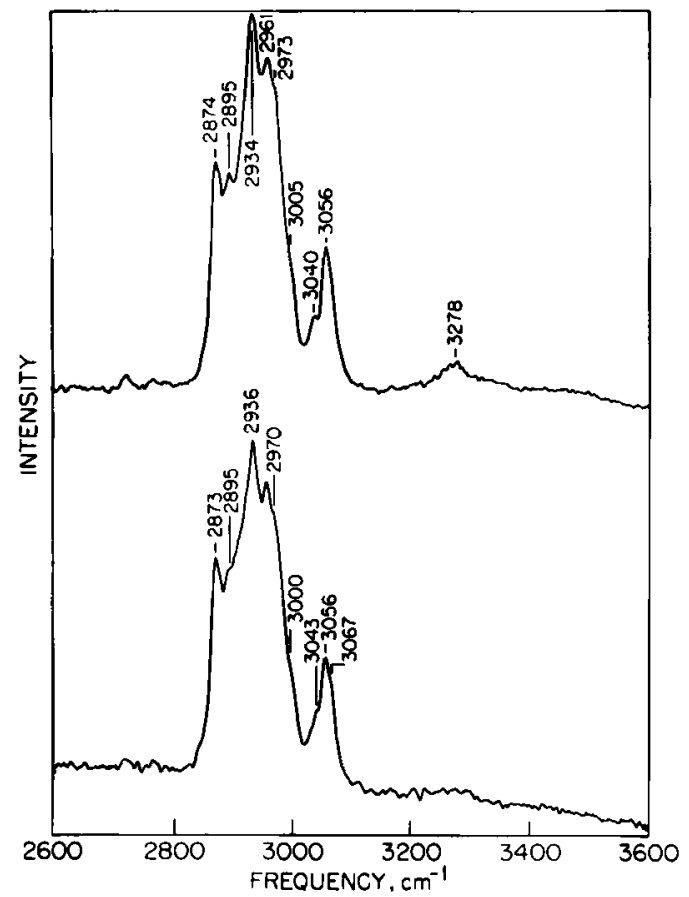

a

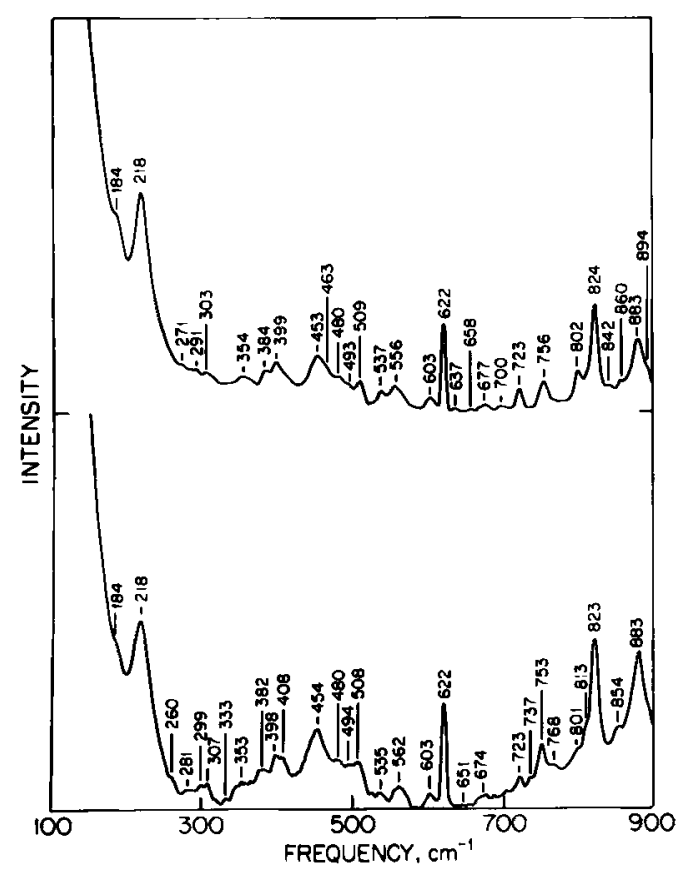

C

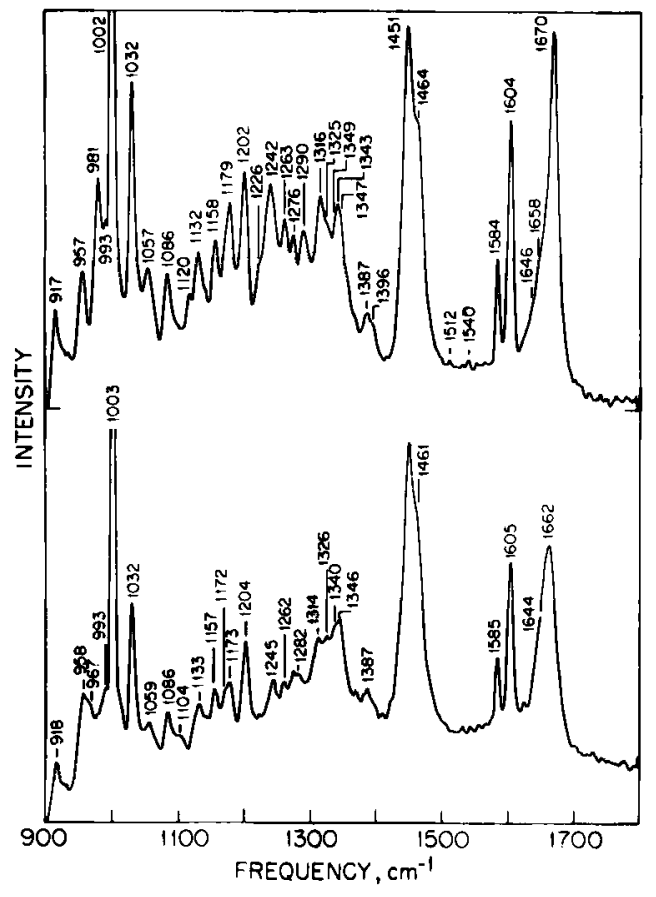

b

\section{FIGURE 2}

Raman spectra of crystalline gramicidin $S$ (upper curve) and N-deuterated gramicidin $S$ (lower curve), (a) $3600-2600 \mathrm{~cm}^{-1}$ region, (b) $1800-900 \mathrm{~cm}^{-1}$ region, (c) $900-100 \mathrm{~cm}^{-1}$ region.

(65) Leu (6) modes of $\mathrm{Ml}$ in Table 2 are calculated at 1655.9 (A) and $1666.0(B) \mathrm{cm}^{-1}$. The intramolecular TDC contribution shifts each of these frequencies by different amounts. In the above case, these contributions are 1.3 and 0.4 $\mathrm{cm}^{-1}$, respectively, leading to intramolecular frequencies of $1667.2(\mathrm{~A})$ and $1666.4(\mathrm{~B}) \mathrm{cm}^{-1}$. Such intramolecular contributions for all of the modes given in Table 2 are as follows [for each structure we give the mixed mode (thus, PF corresponds to the Pro(39) Phe(21) mode) followed by the contributions to the $A$ and $B$ species] : M1-PF: 10.1, 9.2; FP: $-9.7,-10.4$; OL: $1.3,0.4$; LO: $-4.1,0.8$; V: $0.0,0.0$. M2PF: $9.3,8.6$; FP: $-9.3,-9.8 ; \mathrm{O}: 1.6,0.5$; L: $-3.6,-0.5 ; \mathrm{V}:-0.2,1.3$. M3-F: 6.0, 5.4; P: $-6.0,-6.7 ; \mathrm{V}: 2.2,-2.2$; OL: $0.0,-4.7$; LO: $2.1,1.9$. As is clear, the intramolecular 


\section{V.M. Naik et al.}
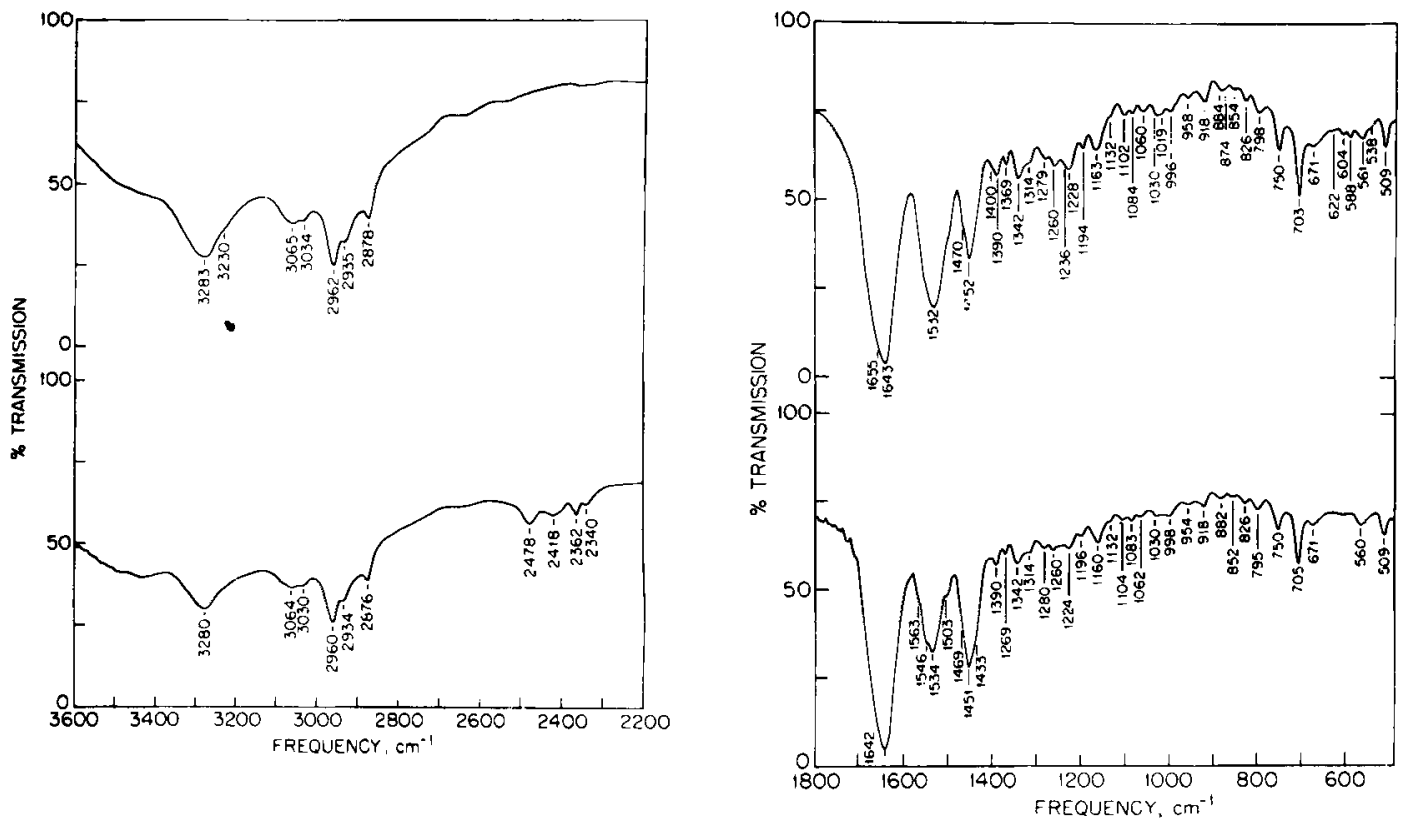

b

FIGURE 3

Infrared spectra of crystalline gramicidin $S$ (upper curve) and $\mathrm{N}$-deuterated gramicidin $\mathrm{S}$ (lower curve). (a) $3600-2200 \mathrm{~cm}^{-1}$ region, (b) $1800-500 \mathrm{~cm}^{-1}$ region.
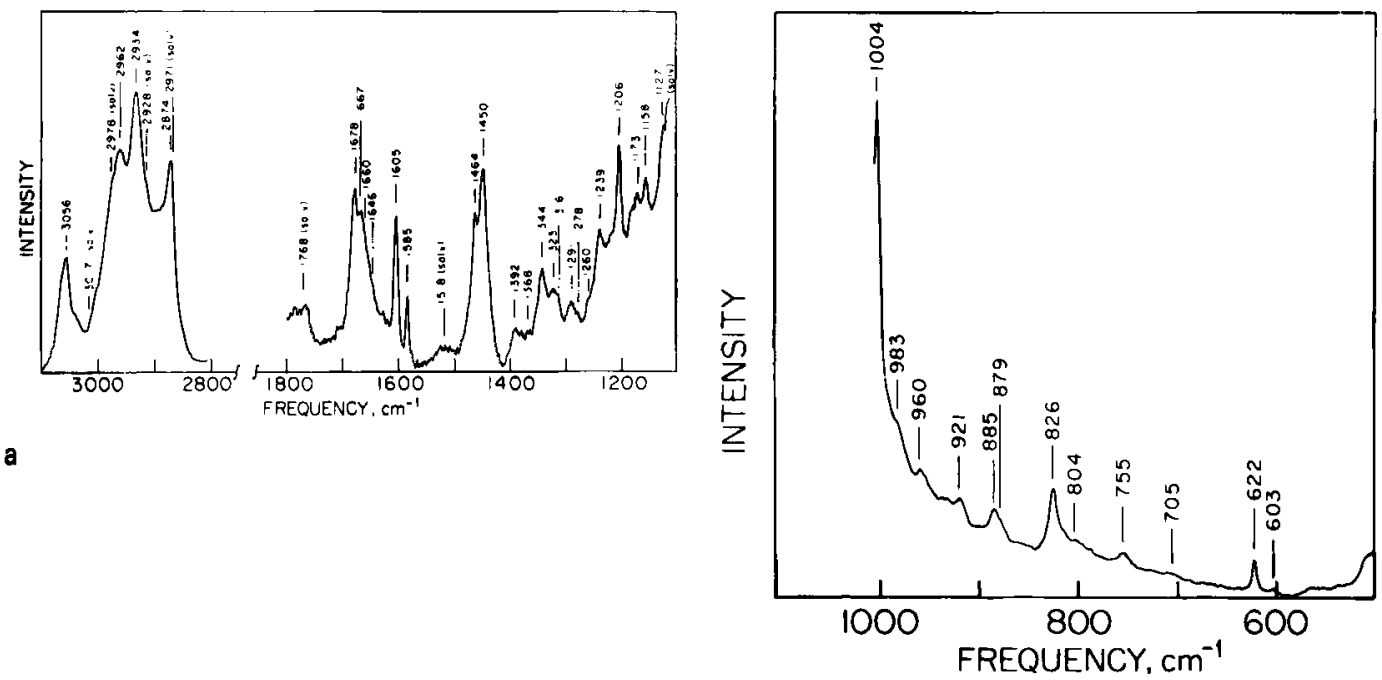

b

FIGURE 4

Raman spectrum of gramicidin $S$ in (a) DMSO-d $d_{6}$ and (b) ms:hanol. 
Vibrational analysis of peptides, polypeptides and proteins

TABLE 2

Amide I modes of gramicidin $S$

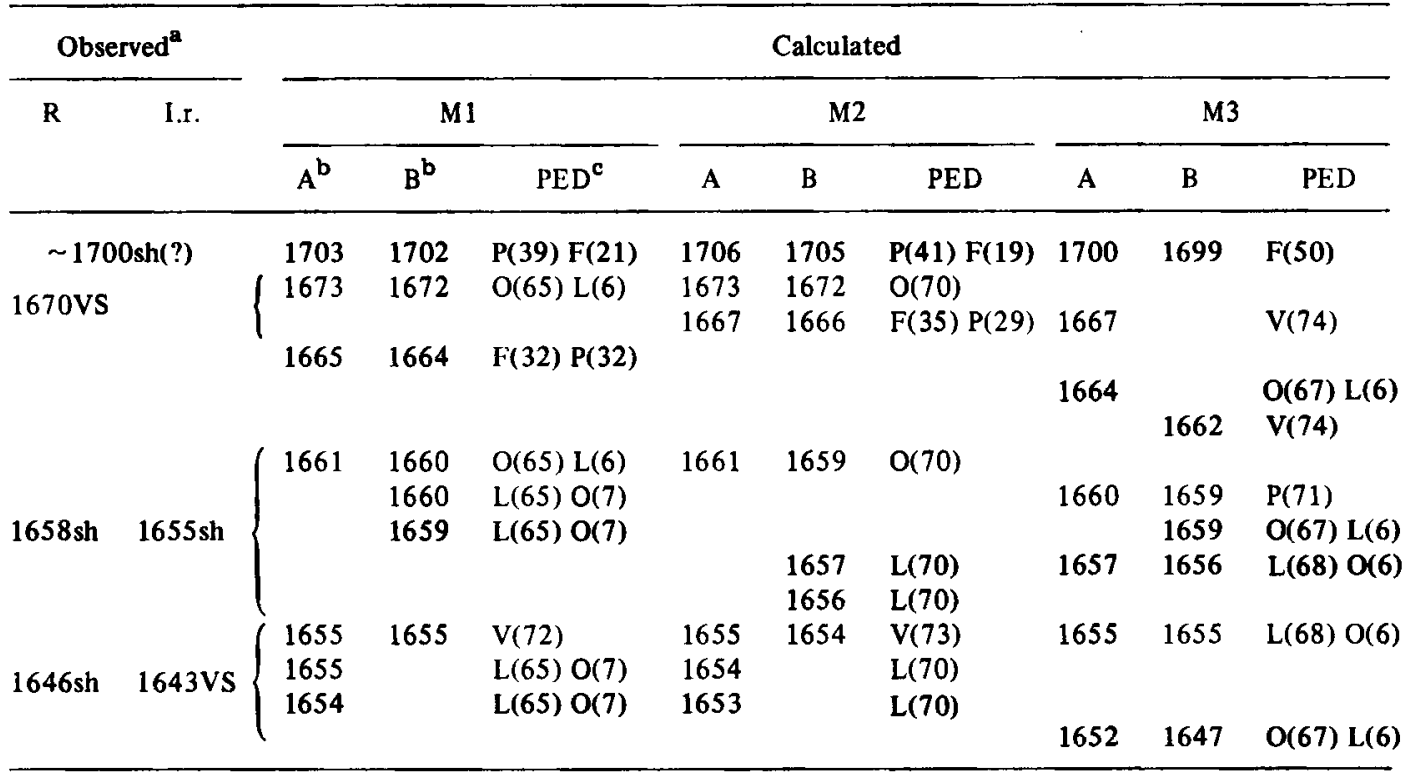

${ }^{a}$ Frequencies in $\mathrm{cm}^{-1}$. VS $=$ very strong, $\mathrm{VW}=$ very weak, $\mathrm{sh}=$ shoulder.

bymmetry species.

${ }^{c}$ PED: potential energy distribution for $\mathrm{CO}$ stretch $\geqslant 5 \%$. Letter represents amino acid residue.

TABLE 3

Amide $I I$ modes of gramicidin $S$

\begin{tabular}{|c|c|c|c|c|c|c|c|c|c|}
\hline \multirow[t]{3}{*}{ I.r. ${ }^{a}$} & \multicolumn{9}{|c|}{ Calculated } \\
\hline & \multicolumn{3}{|c|}{ M1 } & \multicolumn{3}{|c|}{ M2 } & \multicolumn{3}{|c|}{ M3 } \\
\hline & $A^{b}$ & $B^{b}$ & PED $^{c}$ & A & B & PED & $\mathbf{A}$ & B & PED \\
\hline \multirow{4}{*}{$1563 W$} & 1590 & 1586 & $L(32) O(8)$ & 1590 & 1585 & $L(32) O(9)$ & & & \\
\hline & 1588 & 1583 & $L(32) O(8)$ & 1587 & 1582 & $L(32) O(9)$ & & & \\
\hline & $(1562$ & 1569 & $O(34) L(6)$ & 1562 & 1571 & $O(30) L(8)$ & 1565 & 1560 & $\Gamma(32) L(8)$ \\
\hline & 1551 & 1559 & $O(34) L(6)$ & 1552 & 1561 & $O(30) L(8)$ & & & \\
\hline \multirow{4}{*}{$1546 \mathrm{M}$} & 1546 & 1543 & $V(37)$ & 1548 & 1545 & $V(34) O(6)$ & 1554 & 1553 & $\mathrm{O}(22) \mathrm{V}(13)$ \\
\hline & 1545 & 1542 & $V(37)$ & 1546 & 1543 & $V(34) O(6)$ & 1548 & 1546 & $O(22) V(13)$ \\
\hline & & & & & & & 1539 & 1535 & $V(18) L(16)$ \\
\hline & & & & & & & 1539 & 1534 & $V(18) L(16)$ \\
\hline \multirow[t]{2}{*}{$1532 S$} & 1522 & 1522 & $F(36) L(9)$ & 1523 & 1523 & $F(37) L(9)$ & 1527 & 1528 & $O(22) L(14) V(9)$ \\
\hline & 1521 & 1522 & $F(36) L(9)$ & 1522 & 1522 & $F(37) L(9)$ & 1520 & 1521 & $O(22) \mathrm{L}(14) \mathrm{V}(9)$ \\
\hline
\end{tabular}

${ }^{a}$ Frequencies in $\mathrm{cm}^{-1} . \mathrm{S}=$ strong, $\mathrm{W}=$ weak.

${ }^{\mathrm{b}}$ Symmetry species.

${ }^{c}$ PED: potential energy distribution of $\mathrm{NH}$ in-plane bend $>5 \%$. Letter represents amino acid residue. 
TABLE 4

Amide III modes of gramicidin $S$

\begin{tabular}{|c|c|c|c|c|c|c|c|}
\hline \multicolumn{2}{|c|}{ Observed $^{\mathrm{a}}$} & \multicolumn{6}{|c|}{ Calculated } \\
\hline \multirow[t]{2}{*}{$\mathbf{R}$} & \multirow[t]{2}{*}{ I.r. } & \multicolumn{2}{|r|}{ M1 } & \multicolumn{2}{|c|}{ M2 } & \multicolumn{2}{|r|}{ M3 } \\
\hline & & $\nu$ & PED $^{\mathbf{b}}$ & $\nu$ & PED & $v$ & PED \\
\hline $1464 V W^{\mathrm{c}}$ & & $\{1451$ & $\begin{array}{l}F(6) \\
P[1467]^{d}\end{array}$ & 1458 & & 1447 & $V(7)$ \\
\hline 1451VS & 1451VS & & $L[1451]$ & & & & \\
\hline & & $\begin{array}{l}1437 \\
1410\end{array}$ & $V(11)$ & $\begin{array}{l}1443 \\
1414\end{array}$ & $V(10)$ & $\begin{array}{l}1429 \\
1413\end{array}$ & $\mathrm{~L}(8)$ \\
\hline & $1400 \mathrm{VW}$ & 1404 & $O(6) F(5)$ & 1407 & $O(8) F(6)$ & 1398 & $O(10)$ \\
\hline $1396 \mathrm{VW}$ & & 1379 & $\mathrm{~L}(5) \mathrm{O}(5)$ & 1382 & $F(5)$ & 1379 & $F(10) L(5)$ \\
\hline $\begin{array}{l}1387 \mathrm{~W} \\
1370 \mathrm{VW} \\
1347 \mathrm{M}\end{array}$ & $\begin{array}{l}1390 \mathrm{~W} \\
1369 \mathrm{~W}\end{array}$ & & $\begin{array}{l}\mathrm{L}, \mathrm{P}[1395,1386] \\
\mathrm{L}[1370] \\
\mathrm{L}[1351]\end{array}$ & & & & \\
\hline $\begin{array}{l}1343 M \\
1340 \mathrm{~W} \\
1325 \mathrm{VW}\end{array}$ & $1342 \mathrm{MW}$ & 1339 & $\begin{array}{l}V(14) \mathrm{O}(5) \\
\mathrm{L}[1345] \\
\mathrm{CH}_{2}(?)\end{array}$ & 1336 & $V(14)$ & 1342 & $V(12)$ \\
\hline $1316 M$ & $1314 \mathrm{~W}$ & 1308 & $\begin{array}{l}F(7) \\
L[1310]\end{array}$ & 1316 & $F(9)$ & 1319 & $F(16)$ \\
\hline & & 1304 & & 1305 & & 1305 & $L(8)$ \\
\hline $1290 M W$ & & $\left\{\begin{array}{l}1297 \\
1288\end{array}\right.$ & $\begin{array}{l}O(6) \\
F(9) L(7)\end{array}$ & $\begin{array}{l}1298 \\
1291\end{array}$ & $\begin{array}{l}O(6) \\
L(10) F(7)\end{array}$ & $\begin{array}{l}1287 \\
1284\end{array}$ & $L(7)$ \\
\hline $1276 \mathrm{MW}$ & $1279 w$ & & $\mathrm{P}[1271]$ & & & & \\
\hline $1262 M W$ & $1260 \mathrm{~W}$ & 1255 & $V(10)$ & 1256 & $V(11)$ & 1247 & $V(17)$ \\
\hline $1242 M$ & $1236 \mathrm{VW}$ & 1237 & $\begin{array}{r}V(8) F(7) \\
L[1241]\end{array}$ & 1238 & $F(7) V(6)$ & 1237 & $O(11)$ \\
\hline $1226 \mathrm{VW}$ & $1228 \mathrm{~W}$ & 1231 & $\begin{array}{l}O(11) \\
L[1223]\end{array}$ & 1231 & $O(11)$ & 1230 & $F(6) L(5)$ \\
\hline & & $\begin{array}{l}1220 \\
1209\end{array}$ & $L(14) \mathrm{O}(9)$ & $\begin{array}{l}1218 \\
1208\end{array}$ & $L(15) O(7)$ & $\begin{array}{l}1219 \\
1212\end{array}$ & $\begin{array}{l}V(12) \mathrm{O}(6) \\
L(16) \mathrm{V}(6)\end{array}$ \\
\hline $1202 \mathrm{M}$ & $1194 W$ & & $F[1207]$ & & & & \\
\hline $\begin{array}{l}1179 M \\
1158 \mathrm{MW}\end{array}$ & $1163 \mathrm{MW}$ & 1165 & $\begin{array}{l}F(5) \\
P[1165]\end{array}$ & 1160 & $F(5)$ & 1164 & \\
\hline
\end{tabular}

${ }^{a}$ Frequencies in $\mathrm{cm}^{-1} . \mathrm{S}=$ strong, $\mathrm{M}=$ medium, $\mathrm{W}=$ weak, $\mathrm{V}=$ very weak.

${ }^{b}$ Potential energy distribution for $\mathrm{NH}$ in-plane bend $\geqslant 5 \%$. Letters represent amino acid residues. Italicized modes have NH in-plane bend as maximum contributor.

Italicized frequencies weaken on $\mathrm{N}$-deuteration.

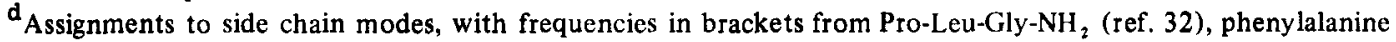
(ref. 33), or polystyrene (ref. 34).

contributions are dependent on the molecular conformation.

The intermolecular TDC contribution, because of the hydrogen bonding through Orn residues, affects only amide I modes of Orn and, because of the admixed contribution, Leu groups. These contributions to the $\mathrm{A}$ and $B$ species are essentially equal, and in the above example amount to $\pm 6.0 \mathrm{~cm}^{-1}$. Thus, we expect the final frequencies to be $1667.2 \pm$ 6.0 (A) and $1666.4 \pm 6.0$ (B) $\mathrm{cm}^{-1}$, giving the values quoted in Table 2 . Other frequencies in the Table were arrived at by similar procedures. We note that in all cases we used for crystalline GrS the intermolecular relationship in the GrSurea complex (15); if this is not applicable, 
TABLE 5

Amide $V$ modes of gramicidin $S$

\begin{tabular}{|c|c|c|c|c|c|c|c|}
\hline \multicolumn{2}{|c|}{ Observed $^{\mathrm{a}}$} & \multicolumn{6}{|c|}{ Calculated } \\
\hline \multirow[t]{2}{*}{$\mathbf{R}$} & \multirow[t]{2}{*}{ I.r. } & \multicolumn{2}{|c|}{ M1 } & \multicolumn{2}{|c|}{ M2 } & \multicolumn{2}{|c|}{ M3 } \\
\hline & & $\nu$ & $\mathrm{PED}^{\mathbf{b}}$ & $\nu$ & PED & $\nu$ & PED \\
\hline $824 M$ & $826 W$ & 831 & $\begin{array}{l}\mathrm{CH}_{2}(?) \\
\mathrm{NH}_{2}(?)\end{array}$ & 830 & & 827 & $O(8)$ \\
\hline \multirow[t]{3}{*}{$802 w^{\mathrm{c}}$} & $798 W$ & & & & & 797 & $O(6)$ \\
\hline & & 786 & & 788 & & 789 & \\
\hline & & 762 & $\begin{array}{l}O(28) \\
F[760]^{d}\end{array}$ & 768 & $O(27)$ & 752 & $F(34)$ \\
\hline $756 \mathrm{MW}$ & $750 \mathrm{MW}$ & 747 & $F(30)$ & 751 & $F(31)$ & 743 & $O(13)$ \\
\hline \multirow[t]{5}{*}{$723 M W$} & & 726 & $F / V(8)$ & 720 & $V(5)$ & 718 & \\
\hline & & 711 & $L(9)$ & 708 & & 707 & \\
\hline & & 706 & $F(5)$ & 702 & $F(8)$ & & \\
\hline & $703 M$ & & $F[700]$ & & & & \\
\hline & & 694 & $V(15)$ & 686 & $V(14)$ & 674 & \\
\hline $675 \mathrm{VW}$ & $671 B$ & 670 & $L(8)$ & 671 & $\mathrm{~L}(5)$ & 666 & \\
\hline $637 V W$ & & 636 & $\mathrm{~L}(8)$ & 633 & & & \\
\hline \multirow[t]{2}{*}{$622 \mathrm{M}$} & $622 \mathrm{VW}$ & & $F[621]$ & & & & \\
\hline & & 622 & & 621 & & 615 & \\
\hline \multirow[t]{2}{*}{$603 W$} & $604 V W$ & 599 & $L(7)$ & 602 & $L(7) V(6)$ & 601 & \\
\hline & $588 \mathrm{VW}$ & 585 & $L(7) O(6)$ & 584 & $L(24)$ & & \\
\hline \multirow[t]{2}{*}{$556 \mathrm{MW}$} & $561 W$ & & $F[558]$ & & & 562 & $V(6)$ \\
\hline & & 540 & & 540 & & 546 & $L(10)$ \\
\hline \multirow[t]{2}{*}{$537 \mathrm{VW}$} & $538 \mathrm{VW}$ & & $P[537]$ & & & 536 & $V(20)$ \\
\hline & & & & & & 527 & $\mathrm{~L}(9)$ \\
\hline $509 \mathrm{~W}$ & $509 \mathrm{MW}$ & & $P[500]$ & & & & \\
\hline
\end{tabular}

${ }^{\mathrm{a}}$ Frequencies in $\mathrm{cm}^{-1} . \mathrm{S}=$ strong, $\mathrm{M}=$ medium, $\mathrm{W}=$ weak, $\mathrm{V}=$ very weak, $\mathrm{B}=$ broad.

${ }^{b}$ Potential energy distribution of NH out-of-plane bend $>5 \%$. Letter represents amino acid residue.

'Italicized frequencies weaken on $\mathbf{N}$-deuteration.

dAssignments to side chain modes, with frequencies in brackets from Pro-Leu-Gly- $\mathrm{NH}_{2}$ (ref. 32), phenylalanine (ref. 33), and polystyrene (ref. 34).

this will modify Orn frequencies near 1672 and $1660 \mathrm{~cm}^{-1}$ for $M 1$ and $M 2$ and near 1664 , 1659 , and $1647 \mathrm{~cm}^{-1}$ for $\mathrm{M} 3$.

The results in Table 2 clearly show that even the unperturbed modes are significantly influenced, in frequency and in mixing, by the conformation of the molecule. Furthermore, the frequencies calculated for the cyclic GrS structure are not a simple superposition of $\beta$ turn and $\beta$-sheet modes, indicating that interactions in such a small molecule are not negligible. For example, for a type II' $\beta$-turn (with, however, Gly in the 3-position rather than Pro) we would expect (23): $\operatorname{CO}(L, V)$ -
1686, $\operatorname{CO}(\mathrm{V}, \mathrm{L})-1673, \operatorname{CO}(\mathrm{F})-1667, \operatorname{CO}(\mathrm{P})-$ 1665; an antiparallel-chain pleated sheet structure should give frequencies near (21) 1694,1669 , and $1632 \mathrm{~cm}^{-1}$, particularly for the Orn residues. In fact, we see that the highest frequency is associated with Phe mixed with Pro in $M 1$ and $M 2$, that $C O(V)$ is much lower, and that no bands are found near 1632 $\mathrm{cm}^{-1}$. This is not really surprising, since it is clear $(30,31)$ that the specific geometry of the TDC interactions determines the final frequencies. But, as can be seen by comparing the PEDs in Table 2 with the above results, the basic mixing of different groups in the normal 
modes of GrS is different than that in the "isolated" $\beta$-turns (23). This illustrates the caution that is necessary in transferring correlations in detail between conformations that are only superficially similar.

The assignments given in Table 2 are based mainly on frequency matching, and can only be suggestive at this stage. They could be made more definite if, for example, spectra of selectively ${ }^{18} \mathrm{O}$ substituted $\mathrm{GrS}$ molecules were available. All three structures predict bands near $1700 \mathrm{~cm}^{-1}$, and the weak shoulders observed in the Raman and i.r. spectra in this region may be assignable to these modes. The very strong Raman band at $1670 \mathrm{~cm}^{-1}$ is reminiscent of a similar band in $\beta$-poly $(L$ alanine) (21) (at $1669 \mathrm{~cm}^{-1}$ ); if it is associated with the $\beta$-sheet-like portion of the $\mathrm{GrS}$ molecule, it would be expected to be most correlated with $A$ species $\mathrm{CO}(\mathrm{V})$ and $\mathrm{CO}(\mathrm{O})$ modes (the others being more closely involved with the $\beta$ turn). It is therefore satisfying that calculated frequencies are found near this value, at 1673 $\mathrm{cm}^{-1}$ for $\mathrm{CO}(0)$ in $\mathrm{M} 1$ and $\mathrm{M} 2$ and at 1667 $\mathrm{cm}^{-1}$ for $C O(V)$ in M3. Since the observed band is a composite one (cf. the broadening and suggestion of splitting on $N$-deuteration), we should expect the calculations to predict at least one other mode in this region. This is clearly the case for $\mathrm{M} 2$ and probably still acceptably the case for $M 1$ and $M 3$. No specific assignments can be given for the weak bands near $1657 \mathrm{~cm}^{-1}$ since there are too many calculated possibilities. The strong i.r. band at $1643 \mathrm{~cm}^{-1}$ should be associated with a B species mode. The frequency agreement is best with the $1647 \mathrm{~cm}^{-1}$ mode of $\mathrm{M} 3$, but it may be difficult to exclude assignments to the $B$ species modes of $\mathrm{Ml}$ and $\mathrm{M} 2$ near $1654 \mathrm{~cm}^{-1}$. The very small apparent shift of the peak of this band on deuteration is difficult to understand, unless it is a consequence of differential shifts of its multiple components.

As a final curious observation, we find that the normal mode calculations for all three structures predict a band near $1480 \mathrm{~cm}^{-1}$ (not shown in Table 2 since it is not strictly an amide I mode) whose largest contribution to the PED for M1 and M2 [next largest for M3] is $F(26)[F(23)$ for $m 3]$. This is an unusually low frequency for a mode with such a large $\mathrm{CO}$ stretch contribution [the other large contributions are $\mathrm{C}^{\alpha} \mathrm{C}(\mathrm{F})$ stretch and $\mathrm{H}^{\alpha}(\mathrm{F})$ bend 2], but it is interesting that a shoulder at 1471 $\mathrm{cm}^{-1}$ in the i.r. definitely shifts down by about $2 \mathrm{~cm}^{-1}$ on deuteration, analogous to the behavior of other $\mathrm{CO}$ stretch modes. If this assignment is indeed correct, it demonstrates again the subtle mixing of internal coordinates that is possible in normal modes and how they can be revealed by such an analysis.

In summary, all three structures give reasonable predictions of the observed amide I bands, and it is difficult to distinguish conclusively between these conformations on the basis of this region.

Amide II region. The amide II [mainly NH inplane-bend (ib)] modes appear as strong bands in the i.r. and weakly or not at all in the Raman spectrum [we find a very weak band near $1540 \mathrm{~cm}^{-1}$ in the Raman spectrum which may be an amide II mode; the 1604 and $1584 \mathrm{~cm}^{-1}$ bands are due to the Phe side chain]. The i.r. spectrum of GrS has a strong asymmetric band centered at $1532 \mathrm{~cm}^{-1}$. On deuteration this band weakens and exhibits distinct amide II components at 1563,1546 , and $1532 \mathrm{~cm}^{-1}$ [the shoulder at $1503 \mathrm{~cm}^{-1}$ is due to the Phe side chain]. In Table 3 we compare these observed bands with the amide II modes calculated for the three structures.

The bands at 1563 and $1546 \mathrm{~cm}^{-1}$ can be well accounted for by all three structures, although the assignments to particular amino acid residues are significantly different in M3 than in $M 1$ and $M 2$. It is interesting to note that, for a standard type II' $\beta$-turn (23), a band corresponding to $\mathrm{NH}(\mathrm{O})$ is predicted at 1559 $\mathrm{cm}^{-1}$, as in $\mathrm{GrS}$, but although a band is also predicted at $1546 \mathrm{~cm}^{-1}$ it corresponds to an NH(L,F) mode rather than the NH(V) mode of GrS $M 1$ and $M 2$ [the corresponding $N H(V)$ mode of the type II $^{\prime} \beta$-turn is predicted (23) at $1531 \mathrm{~cm}^{-1} \mathrm{l}$. The assignment of the strong $1532 \mathrm{~cm}^{-1}$ i.r. band cannot be made with certainty at present: its frequency is in better agreement with those calculated for M3, but an assignment to $\mathrm{M} 1$ or $\mathrm{M} 2$ may not be exclud. able at this point, particularly if, as in the case of other $\beta$-turn structures $(2,3,32)$, there is a tendency to calculate the lower amide II 
frequencies on the low side. This point will. require further study.

Again, the amide II region. of GrS provides no clear-cut distinction between the three structures, although the observed bands can be accounted for quite well by the calculated frequencies.

Amide IIT region. The amide III mode usually involves $\mathrm{NH} \mathrm{ib}$ and $\mathrm{CN}$ stretch coordinates. This mode has been used an an indicator of conformation, although it has been noted that its frequency exhibits a significant sensitivity to side-chain composition (35). In addition, malyses of the normal modes of $\beta$-turns (22, 23) have emphasized that NH ib contributes importantly to many bands in the region of $1200-1400 \mathrm{~cm}^{-1}$ besides those few generally held to be characteristic of conformation. In what follows, we shall examine this entire deuteration-sensitive region.

In Table 4, we list all of the observed Raman and i.r. bands in the frequency region involving NH ib, italicizing those that weaken on deuteration. Those bands that are unaffected by deuteration are very satisfactorily assigned to modes of the Pro (32), Leu (32) (and, by extension, Val), and Phe $(33,34)$ side chains, and the related frequencies in Pro-Leu-Gly$\mathrm{NH}_{2}$ (32) are given in brackets. The deuterationsensitive bands (as well as a few others) mostly contain $\mathrm{H}^{\alpha}$ bend as the major contributor to the PED; we have not listed these but only the NH ib contribution, with the PED percentage in parentheses. In some cases this contribution is in fact the largest, and these have been italicized in the PED column. It is interesting that contributions have to be considered at the $5-10 \%$ level in order to explain the observed deuteration behaviour of some bands.

The first unusual feature to be seen from Table 4 is the extensive range over which NH $\mathrm{ib}$ is predicted to contribute, viz. 1451-1165 $\mathrm{cm}^{-1}$ (for M1). This is much larger than that in Pro-Leu-Gly-NH $\mathrm{NH}_{2}$ (32), viz. $1375-1241 \mathrm{~cm}^{-1}$, but is comparable to that in polyglycine I (29), viz. $1422-1157 \mathrm{~cm}^{-1}$ (for which there are corresponding observed bands in both compounds). Second, not only do observed deuteration-sensitive bands span this range, but there is also very good agreement between observed and calculated frequencies. [The weak Raman band at $1120 \mathrm{~cm}^{-1}$ that disappears on deuteration is probably an $\mathrm{NH}_{2}$ rock mode, which occurs as a strong Raman band at $1135 \mathrm{~cm}^{-1}$ in Pro-Leu-Gly- $\mathrm{NH}_{2}$ (32).] And finally, there seems to be a greater sensitivity to conformation than was the case for the amide I and II modes. Thus, the M2 structure does not predict an NH ib mode (at the $\geqslant 5 \%$ level) near the observed $1464 \mathrm{~cm}^{-1}$ band; $M 3$ is poorer in predicting the value of the $1261 \mathrm{~cm}^{-1}$ bands; and M3 misses badly in accounting for the clearly deuteration-sensitive band at 1179 $\mathrm{cm}^{-1}$ [note the nearly "interna] reference" Phe band at $\left.1202 \mathrm{~cm}^{-1}\right]$, instead predicting such modes in the $1210-1220 \mathrm{~cm}^{-1}$ region where in fact no Raman or i.r. bands are even observed.

These results indicate that the force field has good predictability and sensitivity to conformation, and suggest that perhaps structure $\mathrm{M} 3$ is disfavored compared to $\mathrm{M} 1$ and $\mathrm{M} 2$.

Amide $V$ region. The amide $\mathrm{V}$ mode usually combines $\mathrm{CN}$ torsion with $\mathrm{NH}$ out-of-planebend (ob). It is a very sensitive indicator of conformation, distinguishing easily not only between $\alpha$-helix and $\beta$-sheet structures but also between $\beta$-turns (23). It might therefore be hoped that analysis of this region would provide more definitive information about the conformation of GrS.

In Table 5, we list all of the observed Raman and i.r. bands in the frequency region involving NH ob, italicizing those that weaken on deuteration. There is some problem with the i.r. bands at 750 and $703 \mathrm{~cm}^{-1}$, since they are contributed to mainly by the Phe ring (34), but if they are compared to the "internal reference" bands at 561 and $509 \mathrm{~cm}^{-1}$ (which remain relatively constant) they definitely decrease slightly in intensity on deuteration. The same is true if we consider the $671 \mathrm{~cm}^{-1}$ band to be the peak of a broad band underlying that region. We have listed in Table 5 all of the calculated frequencies in this region, but have given only the $\mathrm{NH}$ ob contributions to these modes.

The most striking feature of the results is that structure M3 cannot account for the significant intensity decreases on deuteration. One of the more clear-cut effects of deuteration 
is the disappearance of i.r. bands at 604 and $588 \mathrm{~cm}^{-1}$; the M3 structure does not even predict modes with $\mathrm{NH}$ ob in this region. Nor does it predict similar intensity decreases at $723,703,671$ and $637 \mathrm{~cm}^{-1}$. The $\mathrm{NH}$ ob coordinate makes no contribution throughout the entire region from 718 to $562 \mathrm{~cm}^{-1}$, but only above and below these frequencies! On the other hand, the $M 1$ and $M 2$ structures can account very well for the deuteration-sensitive bands. (The band near $800 \mathrm{~cm}^{-1}$ that weakens on deuteration is probably an $\mathrm{NH}_{2}$ mode.) The only small difference is that $\mathrm{M} 2$ does not account for the deuteration sensitivity of the $637 \mathrm{~cm}^{-1}$ band.

It seems reasonable, therefore, to conclude that the Raman and i.r. spectra of crystalline GrS, together with the normal mode calculations, argue strongly against the M3 structure but are in good agreement with predictions for M1 and M2, perhaps slightly favoring M1.

\section{Gramicidin $S$ in solution}

The Raman spectrum of GrS in solution (Fig. 4) clearly shows many similarities to that in the crystalline state, suggesting a probable retention of structure in solution. In the brief analysis that follows we examine whether such a hypothesis is consistent with the observed data.

Since DMSO is only a hydrogen bond acceptor, we expect those amide I modes to be most affected whose contributing $\mathrm{CO}$ groups are normally externally hydrogen bonded. These are Phe, Pro, and Orn modes, and we would expect the Orn splitting due to TDC interactions between adjacent molecules in the crystal to disappear in solution. The strong Raman band at $1670 \mathrm{~cm}^{-1}$ in the crystal exhibits two definite peaks in solution, at 1678 and $1667 \mathrm{~cm}^{-1}$. Since $N$-deuteration showed the crystalline band to have two components, it is reasonable to suppose that these components are no longer overlapping in solution. In terms of the M1 structure and the TDC interaction, the Orn mode should be lowered, and therefore the $1667 \mathrm{~cm}^{-1}$ band should be assigned to Orn. This is exactly the frequency we predicted for the Orn amide I mode in the absence of the intermolecular TDC interaction (see above). This implies (cf. Table 2) that the $1678 \mathrm{~cm}^{-1}$ band derives from a Phe mode that has shifted up by about $13 \mathrm{~cm}^{-1}$ from 1665 $\mathrm{cm}^{-1}$, which is in the right direction for a $\mathrm{CO}$ group that is no longer hydrogen bonded to NH. [The quantitative details of such shifts are obviously complicated by unknown di-

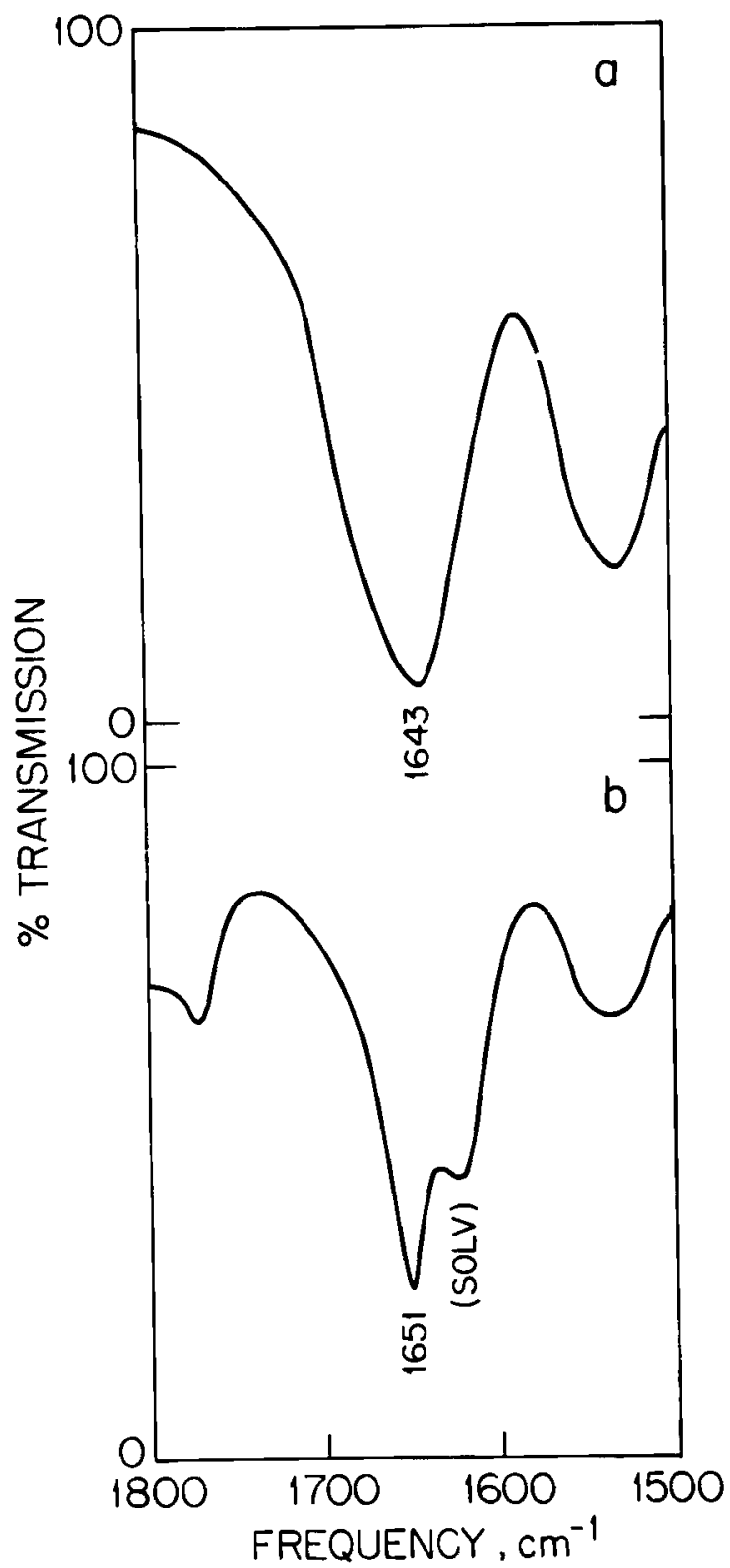

FIGURE 5

Infrared spectra of gramicidin $S$ (a) in the solid state and (b) in DMSO-d 6 
electric constant effects associated with the solvent.] By analogous reasoning, amide I modes of internally hydrogen bonded Leu and Val should be less affected if the molecular conformation in the crystal is retained in solution. This is observed for the relatively unchanged shoulder near $1658 \mathrm{~cm}^{-1}$, which is assigned (cf. Table 2) to Leu. The strong i.r. band at $1643 \mathrm{~cm}^{-1}$ shifts up by $8 \mathrm{~cm}^{-1}$ in DMSO (see Fig. 5). Since this band sharpens and becomes more symmetrical in DMSO [again indicating that the $1655 \mathrm{~cm}^{-1}$ shoulder in the i.r. has not shifted] the results suggest that the Val CO group is somewhat affected, perhaps as a result of a greater accessibility to solvent or a slight change in conformation in this region of the molecule. The integrity of the $\beta$-turn region would result in a relatively constant Leu amide I mode. The behavior of the amide I region is thus consistent with the essential retention of the crystalline conformation in DMSO solution, with a possible small change in the $\mathrm{CO}(\mathrm{Val})$ region.

A similar conclusion is reached from analysis of the amide III modes. In this case, external NH groups can form hydrogen bonds to DMSO, and we therefore do not expect any major changes in frequency if the conformation remains the same, particularly since most modes do not have $\mathrm{NH}$ ib as the major contributor. For those that do have a major NH ib contribution, viz. observed Raman bands at 1343 and $1262 \mathrm{~cm}^{-1}$, it is interesting to note (cf. Table 4) that these correspond to internal NH(Val) hydrogen bonds. We expect such bands to remain relatively constant if the conformation at the $\beta$-turn is unchanged, and indeed the above two bands hardly shift in DMSO (cf. Fig. 4). The $1316 \mathrm{~cm}^{-1}$ Phe band shifts slightly (to $1318 \mathrm{~cm}^{-1}$ ), which may account for the small shift of the $1242 \mathrm{~cm}^{-1}$ band (to $1237 \mathrm{~cm}^{-1}$ ) since it contains a Phe contribution. Thus, the amide III region also indicates an intact $\beta$-turn region.

Finally, this argument is strongly supported by the amide $\mathrm{V}$ region (although here the spectra are for GrS in methanol). The normal mode analysis (cf. Table 5) shows that the weak Raman band at $603 \mathrm{~cm}^{-1}$ should be assigned in M1 to a mode with Leu NH ob. The fact that this band is present in solution strongly suggests that the molecular conformation is essentially unaltered in solution.

\section{CONCLUSIONS}

The normal mode analyses of the low energy M1, M2, and M3 conformations of GrS (12) have demonstrated two important points. First, because of its poorer agreement with observed i.r. and Raman spectra, the M3 structure (of highest energy of the three) can be confidently eliminated as the one present in crystalline GrS. It is more difficult to distinguish between $M 1$ and $M 2$, but this is not unexpected since the backbone structures are so similar. Second, the observed amide I, II, III, and V modes are remarkably well reproduced by the calculation, not only with respect to frequencies but also with regard to the complex forms of the normal modes (cf. the at least nine amide III modes predicted and experimentally shown to have NH ib contributions). Such agreement has also been observed recently in the analysis of Pro-Leu-Gly- $\mathrm{NH}_{2}$ (32), and exhibits the power of the normal mode approach in studying polypeptide structure from vibrational spectra (1). Finally, we note that this analysis provides a strong basis for inferring that the conformation of GrS in the crystal is essentially retained in DMSO and $\mathrm{CH}_{3} \mathrm{OH}$ solutions.

\section{ACKNOWLEDGMENTS}

This research was supported by NSF grants PCM8214064 and DMR-8303610 (V.M.N. and S.K.) and PCM-7920279 (H.A.S.), and by NIH grants GM24893 and AG-00322 (H.A.S. and G.N.)

\section{REFERENCES}

1. Krimm, S. (1983) Biopolymers 22, 217-225

2. Maxfield, F.R., Bandekar, J., Krimm, S., Evans, D.J., Leach, S.J., Nemethy, G. \& Scheraga, H.A. (1981) Macromolecules 14, 997-1003

3. Bandekar, J., Evans, D.J., Krimm, S., Leach, S.J., Lee, S. McQuie, J.R., Minasian, F., Nemethy, G., Pottle, M.S., Scheraga, H.A., Stimson, E.R. \& Woody, R.W. (1982) Int. J. Peptide Protein Res. 19, 187-205 


\section{V.M. Naik et al.}

4. Schmidt, G.M.J., Hodgkin, D.C. \& Oughton, B.M. (1957) Biochem. J. 65, 744-750

5. Hodgkin, D.C. \& Oughton, B.M. (1957) Biochem. J. 65, 752-756

6. Schwyzer, R. (1958) in Amino Acids and Pep. tides with Antimetabolic Activity (Wolstenholme, G.E.W., ed.), p. 171, Churchill, London

7. Venkatachalam, C.M. (1968) Biopolymers 6, 1425-1436

8. Stern, A., Gibbons, W.A. \& Craig, L.C. (1968) Proc. Natl. Acad. Sci. US 61, 734-741

9. Momany, F.A., Vanderkooi, G., Tuttle, R.W. \& Scheraga, H.A. (1969) Biochemistry 8, 744746

10. Ovchinnikov, Y.A., Ivanov, V.T., Bystrov, V.F., Miroshnikov, A.I., Shepel, E.N., Abdullaev, N.D., Efremov, E.S. \& Senyavina, L.B. (1970) Biochem. Biophys Res. Commun, 39, 217-225

11. DeSantis, P. \& Liquori, A.M. (1971) Biopolymers 10, 699-710

12. Dygert, M., Gō, N. \& Scheraga, H.A. (1975) Macromolecules 8, 750-761

13. Rackovsky, S. \& Scheraga, H.A. (1980) Proc. Natl. Acad. Sci. US 77,6965-6967

14. Nemethy, G. \& Scheraga, H.A. (1984) Biochem. Biophys. Res. Commun. 118, 643-647

15. Hull, S.E., Karlsson, R., Main, P., Woolfson, M.M. \& Dodson, E.J. (1978) Nature 275, 206207

16. Abbott, N.B. \& Ambrose, E.J. (1953) Proc. Roy. Soc. (London) A219, 17-32

17. Balasubramanian, D. (1967) J. Am. Chem. Soc. 89, 5445-5449

18. Ingwall, R.T., Gilon, C. \& Goodman, M. (1975) J. Am. Chem. Soc. 97, 4356-4362

19. Kraus, E.M. \& Chan, S.I. (1982) J. Am. Chem. Soc. 104, 1824-1830

20. Fox, J.A., Tu, A.T., Hruby, V.J. \& Mosberg, H.I. (1981) Arch. Biochem. Biophys. 211, 628-631
21. Dwivedi, A.M. \& Krimm, S. (1982) Macromolecules 15, 186-193; (1983) Macromolecules 16,340

22. Bandekar, J. \& Krimm, S. (1979) Proc. Natl. Acad. Sci. US 76, 774-777

23. Krimm, S. \& Bandekar, J. (1980) Biopolymers 19, 1-29

24. Bantlekar, J. \& Krimm, S. (1980) Biopolymers 19, 31-36

25. Maxfield, F.R. \& Scheraga, H.A. (1977) Biochemistry 16, 4443-4449

26. Scheule, R.K., Van Wart, H.E., Vallee, B.L. \& Scheraga, H.A. (1977) Proc. Natl. Acad. Sci. US 74, 3273-3277

27. Dwivedi, A.M. \& Krimm, S. (1984) J. Phys. Chem. 88, 620-627

28. Moore, W.H. \& Krimm, S. (1976) Biopolymers 15, 2439-2464

29. Dwivedi, A.M. \& Krimm, S. (1982) Macromolecules 15, 177-185

30. Krimm, S. \& Abe, Y. (1972) Proc. Natl. Acad. Sci. US 69, 2788-2792

31. Moore, W.H. \& Krimm, S. (1975) Proc. Natl. Acad. Sci. US 72, 4933-4935

32. Naik, V.M. \& Krimm, S. (1984) Int. J. Peptide Protein Res. 23, 1-24

33. Lord, R.C. \& Yu, N.-T. (1970) J. Mol. Biol. 50, 509-524

34. Krimm, S. (1960) Adv. Polymer Sci. 2, 51-172

35. Hsu, S.L., Moore, W.H. \& Krimm, S. (1976) Biopolymers 15, 1513-1528

Address:

Professor S. Krimm

The University of Michigan

Institute of Science and Technology

Biophysics Research Division

2200 Bonisteel Boulevard

Ann Arbor, Michigan 48109

USA 